\title{
1-P-105 Poster Sessions
}

\section{Characterization of pre-incubation inhibitory effects of JPH203 on L-type amino acid transporter 1 function.}

\author{
Kentaro Okunushi ${ }^{1,2}$, Tomomi Furihata ${ }^{1}$, Kosuke Higuchi ${ }^{1}$, Yoshie Reien ${ }^{1}$, Kiyoshi Nakagawa ${ }^{1}$, \\ Naoki Simojo ${ }^{2}$, Naohiko Anzai ${ }^{1}$
}

${ }^{I}$ Dept. Pharm., Grad. Sch. Med., Chiba Univ., ${ }^{2}$ Dept. Pediatrics, Grad. Sch. of Med., Chiba Univ.

BACKGROUND/AIM: JPH203 is a novel anti-cancer drug targeting L-type amino acid transporter 1 (LAT1), which plays a key role in essential amino acid uptake in cancer cells. While the co-incubation inhibitory effect of JPH203 has been characterized in conventional uptake assay, its pre-incubation inhibitory effects remain undetermined. Therefore, we aimed to characterize pre-incubation inhibitory effects of JPH203 on LAT1 function. METHODS: Pre-incubation effects were examined by leucine uptake assay using LAT1-positive human colon cancer HT-29 cells. RESULTS: In time-dependency analysis, pre-incubation of HT-29 cells with $10 \mathrm{uM} \mathrm{JPH203} \mathrm{for} \mathrm{30,} \mathrm{60,} \mathrm{and} 120$ min resulted in a significant decrease of the leucine uptake activity $(42 \%, 32 \%$, and $28 \%$ of those obtained from the control cells, respectively). Similarly, in concentration-dependency analysis, pre-incubation of the cells with JPH203 (1, 10, and $30 \mathrm{uM}$ for $120 \mathrm{~min}$ ) decreased the activity level to $68 \%, 25 \%$, and $3 \%$ of those of the control cells, respectively. CONCLUSION: We have identified potent pre-incubation inhibitory effects of JPH203 on LAT1 function. Combination effects of pre- and co-incubation inhibitory effects are currently under examination. 